\title{
Species delimitation and life stage association of Propsilocerus Kieffer, 1923 (Diptera, Chironomidae) using DNA barcodes
}

\author{
Hai-Jun Yu', Xiao-Long Lin², Rui-Lei Zhang ${ }^{3}$, Qian Wang', Xin-Hua Wang ${ }^{2}$ \\ I Key Laboratory of Aquatic-Ecology and Aquaculture of Tianjin, College of Fishery, Tianjin Agricultural \\ University, Tianjin, 300384, China 2 College of Life Sciences, Nankai University, Tianjin, 300071, China \\ 3 College of Fisheries and life Science, Shanghai Ocean University, Shanghai, 201306, China \\ Corresponding author: Qian Wang (wqgt1999@163.com)
}

Academic editor: Fabio L. da Silva | Received 25 May 2020 | Accepted 8 September 2020 | Published 12 October 2020

http://zoobank.org/43C43D7E-BCA1-47DE-9848-981EB880E938

Citation: Yu H-J, Lin X-L, Zhang R-L, Wang Q, Wang X-H (2020) Species delimitation and life stage association of Propsilocerus Kieffer, 1923 (Diptera, Chironomidae) using DNA barcodes. ZooKeys 975: 79-86. https://doi. org/10.3897/zookeys.975.54668

\begin{abstract}
The utility of COI DNA barcodes in species delimitation is explored as well as life stage associations of five closely related Propsilocerus species: Propsilocerus akamusi (Tokunaga, 1938), Propsilocerus paradoxus (Lundström, 1915), Propsilocerus saetheri Wang, Liu et Paasivirta, 2007, Propsilocerus sinicus Sæther et Wang, 1996, and Propsilocerus taihuensis (Wen, Zhou et Rong, 1994). Results revealed distinctly larger interspecific than intraspecific divergences and indicated a clear "barcode gap". In total, 42 COI barcode sequences including 16 newly generated DNA barcodes were applied to seven Barcode Index Numbers (BINs). A neighbor-joining (NJ) tree comprises five well-separated clusters representing five morphospecies. Comments on how to distinguish the larvae of $P$. akamusi and P. taihuensis are provided.
\end{abstract}

\section{Keywords}

barcode gap, bioindicator, chironomid, COI, genetic distance, larval association, Propsilocerus taihuensis

Copyright Hai-Jun Yu et al. This is an open access article distributed under the terms of the Creative Commons Attribution License (CC BY 4.0), which permits unrestricted use, distribution, and reproduction in any medium, provided the original author and source are credited. 


\section{Introduction}

The genus Propsilocerus Kieffer, 1923 (Fig. 1) was erected with the type species Propsilocerus lacustris Kieffer, 1923. At present, there are nine Propsilocerus species described in the Palaearctic and Oriental regions (Sæther and Wang 1996; Zelentsov 2000; Tang et al. 2004; Wang et al. 2007; Makarchenko and Makarchenko 2009) and one unnamed species from the Nearctic region (Cranston et al. 2011). Larvae of Propsilocerus usually inhabit eutrophic rivers and lakes. Because of their great densities and ability to adapt to different freshwater bodies, they are important food items for fishes and birds, and also bioindicators in monitoring of the freshwater ecosystem. However, the high morphological similarity between closely related species within Propsilocerus and intraspecific morphological variation have likely led to misidentifications, particularly in larvae. The morphological diagnosis (e.g., AR, $\mathrm{LR}_{1}$ ) of closely related morphospecies needs to be evaluated to verify the identity of each Propsilocerus species.

All four common species, Propsilocerus akamusi (Tokunaga, 1938), Propsilocerus paradoxus (Lundström, 1915), Propsilocerus sinicus Sæther et Wang, 1996, and Propsilocerus taihuensis (Wen, Zhou et Rong, 1994) are present in Yuqiao Reservoir, Jizhou Distinct, Tianjin, China during the spring and autumn. As a result, larvae of these four species usually have been misidentified as Propsilocerus akamusi by ecologists in China.

DNA barcodes (Hebert et al. 2003a, b) have proven successful in species delimitation and recognition of cryptic species diversity in chironomids (Anderson et al. 2013; Lin et al. 2015; Lin et al. 2018; Song et al. 2018). However, only one named (P. akamusi) and one unidentified species (Propsilocerus sp. JC-2015) have registered public DNA barcodes in the Barcode of Life Data systems (BOLD) (Ratnasingham and Hebert 2007). Thus, it is necessary to barcode more Propsilocerus species, which are common species in polluted rivers and lakes.

Here we explore the utility of DNA barcodes in species delimitation and in associating life stage in Propsilocerus. Registering new barcodes of Propsilocerus species will improve the reference library of Chironomidae (Ekrem et al. 2007) for DNA metabarcoding in biodiversity assessment in monitoring freshwater ecosystems.

\section{Materials and methods}

In this study, 42 specimens of five Propsilocerus species (P. akamusi, P. paradoxus, P. saetheri, P. sinicus, and P. taihuensis) from China, Japan, Norway, and South Korea with COI barcodes were included. Twenty-six specimens with public COI barcodes were retrieved from BOLD and GenBank, and an additional 16 individuals of four Propsilocerus species were collected from the eutrophic lakes and reservoirs from Hebei Province, Shanghai and Tianjin, China, using D-nets, sweep nets, and light traps.

Larvae were preserved in $95 \%$ ethanol, adults in $85 \%$ ethanol, and stored at $4{ }^{\circ} \mathrm{C}$ in the dark before morphological and molecular studies. Photographs of all intact specimens were taken before dissection using a ZEISS camera mounted on a ZEISS stereomicroscope using the software AxioVision Rel. 4.8. at the College of Life Sciences, 


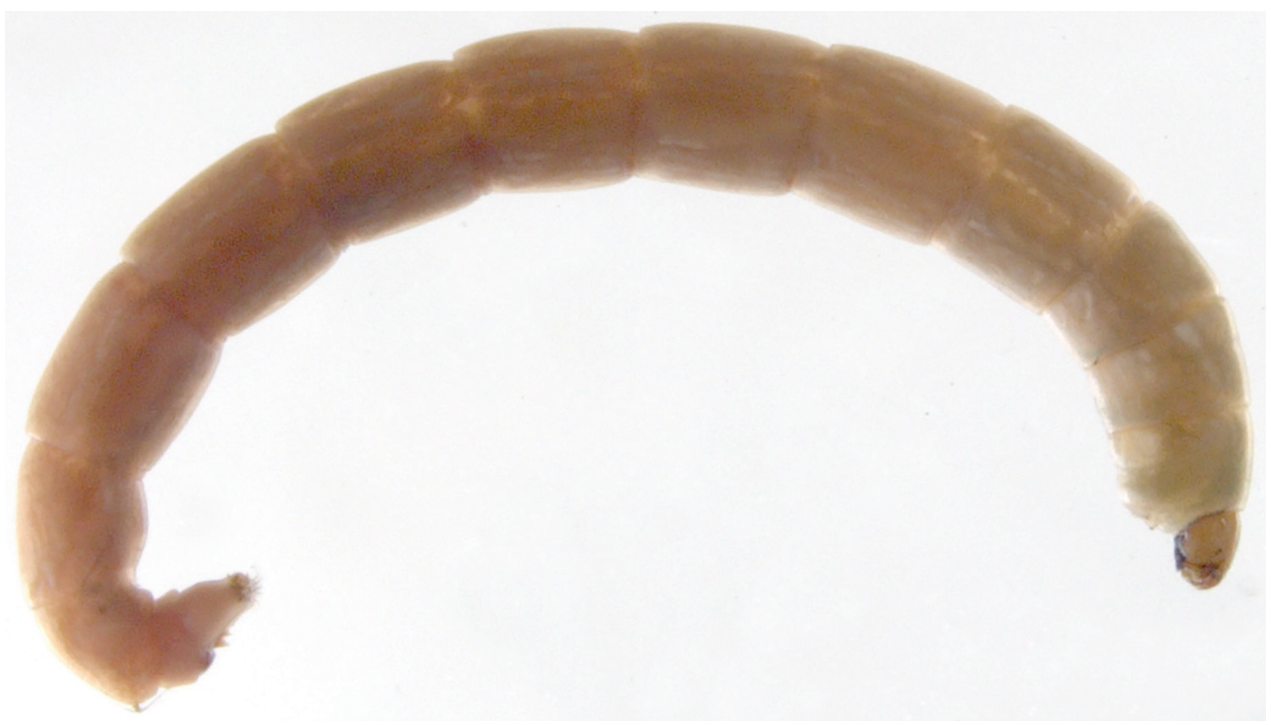

Figure I. Larva of Propsilocerus taihuensis (Wen, Zhou \& Rong, 1994).

Nankai University, Tianjin, China. Digital photographs of slide specimens were taken at 300-dpi resolution using a Nikon Digital Sight DS-Fil camera mounted on a Nikon Eclipse 80i compound microscope.

Extraction of genomic DNA was done following the standard protocol of the Qiagen DNeasy Blood \& Tissue Kit, except the volume of DNA template was $110 \mu \mathrm{l}$ in the final step. Morphological terminology used in this work is according to Sæther (1980). The cleared exoskeleton of adults was mounted in Euparal on microscope slides together with the corresponding wings, legs, and antennae after DNA extraction. Voucher specimens from China were deposited in the College of Life Sciences, Nankai University, Tianjin, China.

DNA amplifications of COI barcode sequences with the universal primers LCO1490 and HCO2198 (Folmer et al. 1994) were carried out at the College of Fishery, Tianjin Agricultural University. Polymerase chain reaction (PCR) was set up using $12.5 \mu \mathrm{l} 2 \times$ Es Taq MasterMix (CoWin Biotech Co., Beijing, China), $0.625 \mu \mathrm{l}$ of each primer, $2.5 \mu \mathrm{l}$ template DNA, and $8.75 \mu \mathrm{ddd}_{2} \mathrm{O}$ to make a total of $25 \mu \mathrm{l}$ for each sample. PCR was performed on a MasterCycler Gradient (Biometra GmbH, Göttingen, Germany), with an initial denaturation step of $95^{\circ} \mathrm{C}$ for 4 min followed by 40 cycles at $94^{\circ} \mathrm{C}$ for $45 \mathrm{~s}, 52{ }^{\circ} \mathrm{C}$ for $45 \mathrm{~s}, 72{ }^{\circ} \mathrm{C}$ for $1 \mathrm{~min}$, and one final extension at $72{ }^{\circ} \mathrm{C}$ for $10 \mathrm{~min}$. PCR products were electrophoresed in $1.5 \%$ agarose gel, purified and sequenced with ABI 3730 (BGI TechSolutions Co., Lit. Beijing, China).

Raw sequences were edited and assembled in SeqMan version 7.1.0 (in the LaserGene package, DNASTAR, Madison, USA), aligned using the Muscle algorithm (Edgar 2004), and checked for stop codons on the amino acids in MEGA version 7.0 (Kumar et al. 2016). Sequences were uploaded on BOLD with collateral information and images. A public dataset including all 42 specimens, "DNA barcodes of Propsilocerus [DS- 
PROPSIL]", can be found in BOLD. The neighbor-joining (NJ) trees were constructed in MEGA using Kimura 2-Parameter (K2P) substitution model, 1000 bootstrap replicates and the "pairwise deletion" option for missing data. The pairwise distances of five Propsilocerus species were calculated using K2P model in MEGA. To detect the "barcode gap", the aligned sequence dataset was subject to Automatic Barcode Gap Discovery (ABGD) (Puillandre et al. 2012) with the K2P model, following the default setting.

\section{Results}

\section{DNA barcode analyses}

In general, the data showed distinctly larger interspecific than intraspecific divergence, and there was a clear "barcode gap" in the pairwise K2P distances (Fig. 2). The minimum interspecific genetic distance between the closely related morphospecies $P$. akamusi and P. taibuensis is $13.4 \%$. The maximum intraspecific distance of $P$. akamusi is $5.2 \%, 3.0 \%$ in $P$. taibuensis, $0.8 \%$ in $P$. paradoxus, and $0.5 \%$ in $P$. saetheri (P. sinicus is a singleton). Examining the present dataset in BOLD, 42 COI barcodes from five morphospecies of Propsilocerus were assigned into seven barcode index numbers (BINs). There are two BINs in each species P. akamusi (BOLD:ACB4994, BOLD:ACQ5058) and $P$. taihuensis (BOLD:ADX1391, BOLD:ADK5547), and a unique BIN in P. paradoxus (BOLD:ADX2356), P. saetheri (BOLD:AAM7072), and P. sinicus (BOLD:ADX6952).

The neighbor-joining tree (Fig. 3) based on 42 COI barcodes of Propsilocerus species revealed five distinct genetic clusters, corresponding to five morphospecies. The unidentified species (Propsilocerus sp. JC-2015) grouped into P. taihuensis (Fig. 3). Lar-

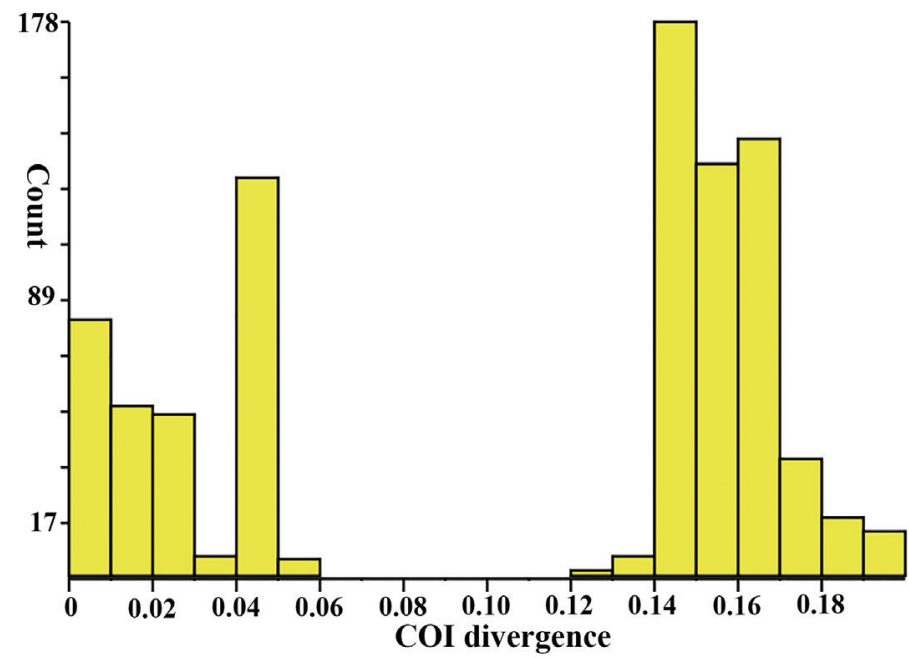

Figure 2. Histogram of pairwise K2P distances of 42 aligned sequences of five Propsilocerus morphospecies. The figure was a result of analysis with ABGD using the K2P model. The horizontal axis shows the pairwise K2P-distance, and the vertical axis shows the number of pairwise sequence comparisons. 


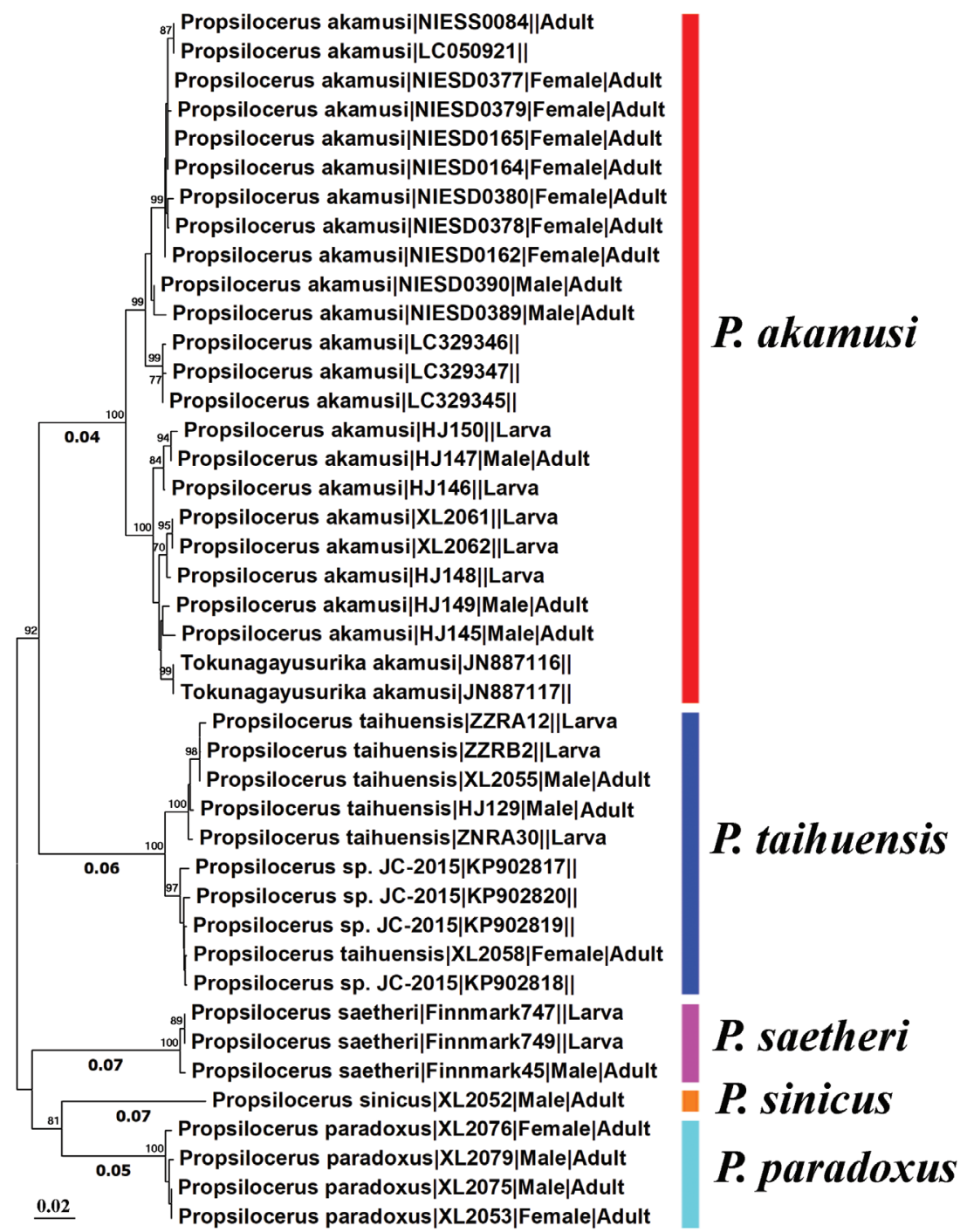

Figure 3. Neighbor-joining tree based on the 42 COI barcode sequences of Propsilocerus. Bootstrap support (1000 replicates) $>70 \%$ are labelled.

vae of $P$. akamusi, $P$. saetheri, and $P$. taihuensis can now be associated with adults based on DNA barcodes.

\section{Morphology}

Although it is feasible to distinguish species of Propsilocerus by referring to the works of Makarchenko and Makarchenko (2009), Sæther and Wang (1996), and Wang et al. (2007), misidentification of the larvae of Propsilocerus often occurs due to high morphological similarities. Currently, larvae of six Propsilocerus named species have 
been described. Tang et al. (2004) provided a key to the larvae of known species, and described the larvae of $P$. taihuensis based on the material from the type locality, Wuli Lake, Taihu Lake, Jiangsu Province, China. However, these larvae of P. taihuensis were not reared, and their identification could be uncertain. In this study, the larvae of P. taihuensis have been associated with adults using DNA barcodes. After reexamining the voucher and type specimens, we confirm that the description of the larvae of P. taihuensis by Tang et al. (2004) is correct, and P. akamusi can be separated from $P$. taihuensis by the relative lengths of the third and fourth antennal segments and the numbers of lateral teeth (Fig. 4) on the mentum (Tang et al. 2004). However, this is difficult in practice because larvae of $P$. akamusi and $P$. taibuensis both have dark head capsules, and 9-10 lateral teeth (often not easy to count) on the mentum, and short third and fourth antennal segments (Fig. 4). These two species can be more ef-
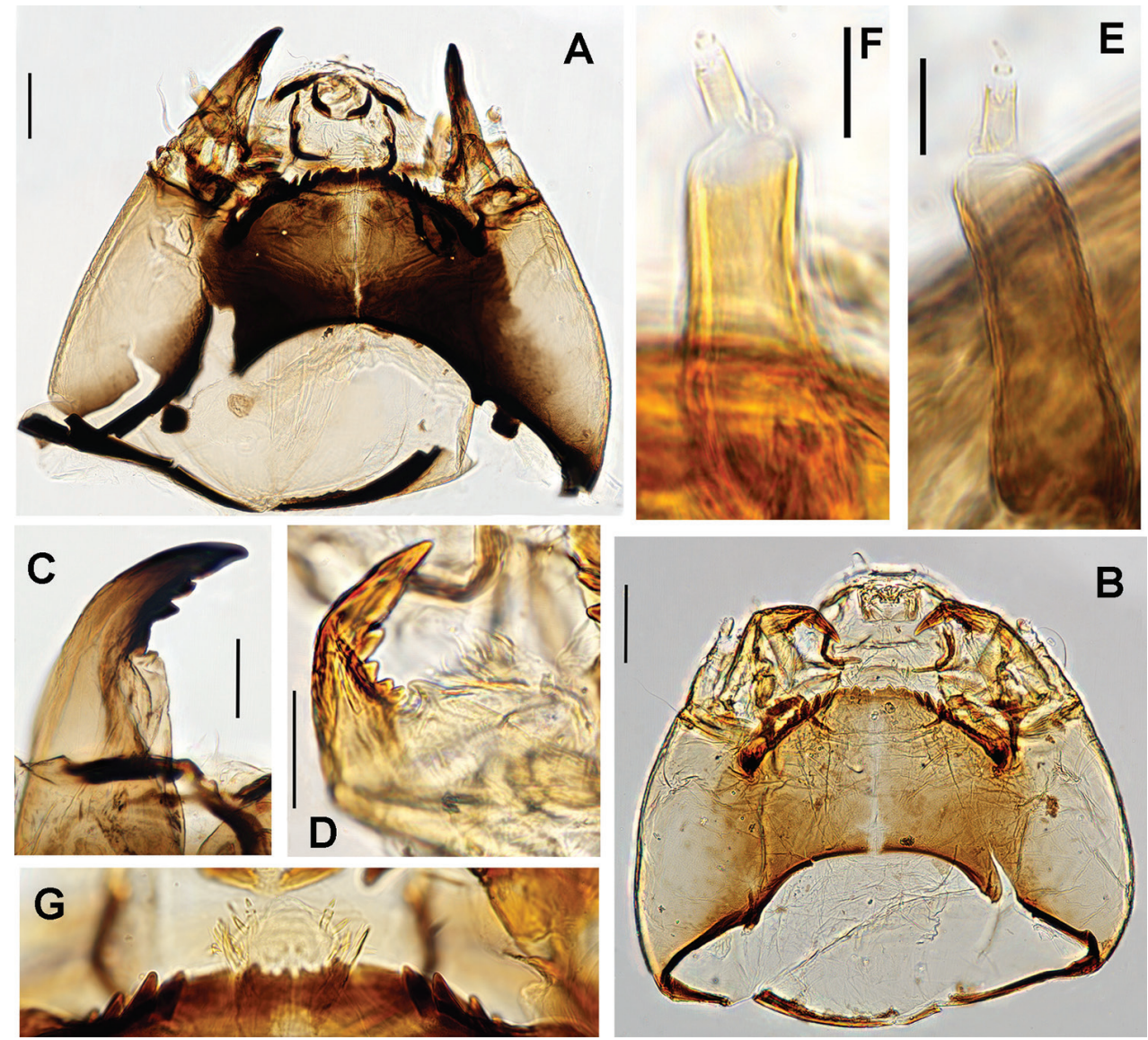

Figure 4. Head capsules of Propsilocerus akamusi (Tokunaga, 1938) and Propsilocerus taihuensis (Wen, Zhou $\&$ Rong, 1994) A head capsule of $P$. akamusi, ventral view $\mathbf{B}$ head capsule of $P$. taihuensis, ventral view $\mathbf{C}$ mandible of $P$. akamusi $\mathbf{D}$ mandible of $P$. taihuensis $\mathbf{E}$ antenna of $P$. akamusi $\mathbf{F}$ antenna of $P$. taihuensis $\mathbf{G}$ prementohypopharyngeal complex of $P$. taihuensis. Scale bar: $100 \mu \mathrm{m}(\mathbf{A}, \mathbf{B}), 50 \mu \mathrm{m}(\mathbf{C}, \mathbf{D}), 25 \mu \mathrm{m}(\mathbf{E}, \mathbf{F})$. 
fectively distinguished by observing the premandible and mentum. In P. akamusi, the premandible is bifid, and the median portion of the mentum with one median notch and is subdivided into small teeth; whereas in P. taibuensis, the premandible is simple, and the median portion of the mentum has four teeth and one median notch.

It was also discovered that the undescribed Nearctic larva (Cranston et al. 2011) is closely related to P. taihuensis (Fig. 4B) in having a well-developed premento-hypopharyngeal complex (Fig. 4G) and the apical tooth longer and pointed, longer than the combined width of four teeth.

\section{Conclusions}

Our study has revealed strong concordance between morphospecies and DNA barcodes of Propsilocerus. Distinct "barcode gaps" were discovered among Propsilocerus species. DNA barcodes have been used to associate different life stages, and the unidentified species (Propsilocerus sp. JC-2015) was confidently assigned to P. taihuensis. Comments on how to distinguish this species from congeners on the larvae of $P$. taihuensis are given.

\section{Acknowledgements}

We thank the following people who have kindly sent us specimens for our study: Dr Wen-Bin Liu (Tianjin Normal University, Tianjin, China) and Tao Zheng (Tianjin Agricultural University, Tianjin, China). Financial support from the National Natural Science Foundation of China $(31672264,31301908,31900344)$ and the China Postdoctoral Science Foundation Grant (2018M640227) are acknowledged with thanks.

\section{References}

Anderson AM, Stur E, Ekrem T (2013) Molecular and morphological methods reveal cryptic diversity and three new species of Nearctic Micropsectra (Diptera: Chironomidae). Freshwater Science 32: 892-921. https://doi.org/10.1899/12-026.1

Cranston PS, Barley E, Langley GE, Dieffenbacher-Krall A, Longmuir A, Zloty J (2011) Propsilocerus Kieffer (Diptera: Chironomidae) from the Nearctic. Aquatic Insects 33: 343-350. https://doi.org/10.1080/01650424.2011.639306

Edgar RC (2004) MUSCLE: multiple sequence alignment with high accuracy and high throughput. Nucleic Acids Research 32: 1792-1797. https://doi.org/10.1093/nar/gkh340

Ekrem T, Willassen E, Stur E (2007) A comprehensive DNA sequence library is essential for identification with DNA barcodes. Molecular Phylogenetics and Evolution 43: 530-542. https://doi.org/10.1016/j.ympev.2006.11.021 
Folmer O, Black M, Hoeh W, Lutz R, Vrijenhoek R (1994) DNA primers for amplification of mitochondrial cytochrome $c$ oxidase subunit I from diverse metazoan invertebrates. Molecular Marine Biology and Biotechnology 3: 294-299.

Hebert PDN, Cywinska A, Ball SL (2003a) Biological identifications through DNA barcodes. Proceedings of the Royal Society of London B: Biological Sciences 270: 313-321. https:// doi.org/10.1098/rspb.2002.2218

Hebert PDN, Ratnasingham S, de Waard JR (2003b) Barcoding animal life: cytochrome $c$ oxidase subunit 1 divergences among closely related species. Proceedings of the Royal Society of London B: Biological Sciences 270: S96-S99. https://doi.org/10.1098/rsbl.2003.0025

Kumar S, Stecher G, Tamura K (2016) MEGA7: Molecular Evolutionary Genetics Analysis version 7.0 for bigger datasets. Molecular Biology and Evolution 33: 1870-1874. https:// doi.org/10.1093/molbev/msw054

Lin X-L, Stur E, Ekrem T (2015) Exploring genetic divergence in a species-rich insect genus using 2790 DNA Barcodes. PloS ONE 10: e0138993. https://doi.org/10.1371/journal. pone. 0138993

Lin X-L, Stur E, Ekrem T (2018) DNA barcodes and morphology reveal unrecognized species of Chironomidae (Diptera). Insect Systematics \& Evolution 49: 329-398. https://doi. org/10.1163/1876312X-00002172

Makarchenko E, Makarchenko M (2009) Propsilocerus amurensis sp. n. (Diptera, Chironomidae, Orthocladiinae) from Amur River basin (Russian Far East). Evraziatskii entomologicheskii zhurnal (Euroasian entomological journal) 8: 261-263.

Puillandre N, Lambert A, Brouillet S, Achaz G (2012) ABGD, Automatic Barcode Gap Discovery for primary species delimitation. Molecular Ecology Notes 21: 1864-1877. https:// doi.org/10.1111/j.1365-294X.2011.05239.x

Ratnasingham S, Hebert PDN (2007) BOLD: The Barcode of Life Data System (www.barcodinglife.org). Molecular Ecology Notes 7: 355-364. https://doi.org/10.1111/j.14718286.2007.01678.x

Sæther OA (1980) Glossary of chironomid morphology terminology (Diptera: Chironomidae). Entomologica Scandinavica Supplement 14: 1-51.

Sæther OA, Wang XH (1996) Revision of the orthoclad genus Propsilocerus Kieffer (equals Tokunagayusurika Sasa) (Diptera: Chironomidae). Entomologica Scandinavica 27: 441479. https://doi.org/10.1163/187631296X00151

Song C, Lin X-L, Wang Q, Wang X-H (2018) DNA barcodes successfully delimit morphospecies in a superdiverse insect genus. Zoological Scripta 47: 311-324. https://doi. org/10.1111/zsc. 12284

Tang HQ, Guo YH, Wang XH, Saether OA (2004) The pupa and larva of Propsilocerus taihuensis (Wen, Zhou \& Rong) (Diptera: Chironomidae), with complete keys to all immatures of the genus Propsilocerus. Aquatic Insects 26: 265-272. https://doi.org/10.1080/0165-0420400000585

Wang XH, Liu YD, Paasivirta L (2007) A new species of Propsilocerus Kieffer from Finland (Diptera: Chironomidae, Orthocladiinae). In: Andersen T (Ed.) Contributions to the Systematics and Ecology of Aquatic Diptera - A tribute to Ole A Sæther. The Caddis Press, Columbus, Ohio, 309-313.

Zelentsov NI (2000) A new Orthocladiin species of the genus Propsilocerus (Diptera, Chironomidae) from Taimyr Peninsula. Zoologichesky Zhurnal 79: 1242-1246. 